\title{
Triggered single-photon emission in the red spectral range from optically excited InP/(AI,Ga)InP quantum dots embedded in micropillars up to $100 \mathrm{~K}$
}

\author{
M. Bommer, ${ }^{1, a)}$ W.-M. Schulz, ${ }^{1}$ R. Roßbach, ${ }^{1}$ M. Jetter, ${ }^{1}$ P. Michler, ${ }^{1}$ T. Thomay, ${ }^{2}$ \\ A. Leitenstorfer, ${ }^{2}$ and R. Bratschitsch ${ }^{2, b)}$ \\ ${ }^{1}$ Institut für Halbleiteroptik und Funktionelle Grenzflächen, University of Stuttgart, D-70569 Stuttgart, \\ Germany \\ ${ }^{2}$ Department of Physics and Center for Applied Photonics, University of Konstanz, D-78457 Konstanz, \\ Germany
}

(Received 1 June 2011; accepted 28 July 2011; published online 21 September 2011)

\begin{abstract}
Systematic excitation power and temperature-dependent measurements on the emission lines of single self-assembled $\mathrm{InP} /\left(\mathrm{Al}_{0.20} \mathrm{Ga}_{0.80}\right)_{0.51} \mathrm{In}_{0.49} \mathrm{P}$ quantum dots embedded in micropillars have been performed. The quantum dots were excited optically via a pulsed laser and their luminescence was collected using a micro-photoluminescence setup. The exciton and biexciton intensity, linewidth, and spectral position was investigated in a temperature range from $4 \mathrm{~K}$ up to $130 \mathrm{~K}$. Singlephoton emission from the quantum dots is presented up to a temperature of $100 \mathrm{~K}$, confirmed by photon-statistics measurements. (C) 2011 American Institute of Physics. [doi:10.1063/1.3633218]
\end{abstract}

\section{INTRODUCTION}

High-quality quantum dot (QD) single-photon (SP) sources $^{1-5}$ are of high interest for quantum cryptography applications due to their easy handling and scalability. However, an operation temperature above liquid nitrogen temperature and ideally in the Peltier-cooling regime is desired for their use in commercial devices.

Several publications dealing with single-photon generation by quantum dots toward high temperatures have been published in the last years. So far, wide-bandgap material systems offer the highest working temperatures for epitaxially grown QDs. Single-photon emission utilizing microphotoluminescence ( $\mu \mathrm{PL})$ has been demonstrated up to $200 \mathrm{~K}$ for $\mathrm{CdSe} / \mathrm{ZnSe}$ (Ref. 6) and for GaN/AIN QDs. ${ }^{7}$ The most studied material systems InAs/GaAs and InGaAs/ $\mathrm{GaAs}$, however, are more limited in their temperature range, due to their typically lower charge carrier confinement potentials that leads to a loss of charge carriers out of the QD at higher temperatures. An additional limiting factor in these material systems is the low biexciton (XX) binding energy, separating exciton $(\mathrm{X})$, and $\mathrm{XX}$ energetically, which results in an overlap of these emission lines at elevated temperatures resulting in lower suppression of multi-photon events.

$\mu$ PL measurements on InGaAs/GaAs QDs show SP emission up to $120 \mathrm{~K}^{8}$ Pyramidal InGaAs/AlGaAs QDs reveal SP emission up to $80 \mathrm{~K}$ with an XX binding energy of $3.2 \mathrm{meV}{ }^{9}$ Single-photon generation from InP QDs has been demonstrated for temperatures up to $80 \mathrm{~K}$ optically pumped ${ }^{10-12}$ and electrically driven. ${ }^{13}$

Nevertheless, self-assembled InP QDs are attractive candidates for SP generation, because they exhibit luminescence in the visible red spectral range within the detection

\footnotetext{
${ }^{a)}$ Electronic mail: m.bommer@ihfg.uni-stuttgart.de. URL: http:www.ihfg. uni-stuttgart.de.

${ }^{b)}$ Present address: Institute of Physics, Chemnitz University of Technology, D-09126 Chemnitz, Germany.
}

maximum of common silicon SP detectors. ${ }^{14}$ Additionally the emission wavelength can be tuned to an absorption minimum of polymer optical fibers (POF). This allows efficient cryptographic communication combined with the ease of inand out-coupling inherent to POF based fiber networks, due to their large core diameters. Also their XX binding energy is relatively large in comparison to InGaAs QDs, ranging from 4 to $7 \mathrm{meV},{ }^{15}$ which lowers the energetic overlap of the $\mathrm{XX}$ and $\mathrm{X}$ emission lines at elevated temperatures.

In our work, InP QD sample structures with aluminum containing barrier layers have been chosen to improve the charge carrier confinement and thus the high temperature operation of non-classical photon emission. ${ }^{16}$ To enhance the photon collection efficiency, the QD layer is additionally sandwiched between distributed Bragg reflectors forming a $\lambda$-cavity. In order to achieve lateral mode confinement, micropillars (MPs) with diameters between 1.2 and $5 \mu \mathrm{m}$ were milled out of the planar cavity. Together with a low spatial dot density, investigation on a single MP provides access to individual energetically separated QDs.

\section{SAMPLE GROWTH AND PREPARATION}

The layer materials were deposited using metal-organic vapor-phase epitaxy (MOVPE) at $750{ }^{\circ} \mathrm{C}$ on a (100)-oriented GaAs:Si substrate tilted by $6^{\circ}$ toward the [111]A direction. As bottom and top distributed Bragg reflectors (DBRs) 45 pairs $\lambda / 4$-periods of $\mathrm{Al}_{0.5} \mathrm{Ga}_{0.5} \mathrm{As}: \mathrm{Si} / \mathrm{AlAs}: \mathrm{Si}$ and $36 \lambda / 4$ thick pairs of $\mathrm{Al}_{0.5} \mathrm{Ga}_{0.5} \mathrm{As}: \mathrm{C} / \mathrm{Al}_{0.95} \mathrm{Ga}_{0.05} \mathrm{As}: \mathrm{C}$ were deposited, respectively, forming a planar cavity. The InP QDs, grown in Stranski-Krastanow growth mode ${ }^{17}$ at $650{ }^{\circ} \mathrm{C}$, are in spatial overlap with the E-field antinode of the fundamental planar cavity mode. A $10 \mathrm{~nm}$ thick $\left(\left(\mathrm{Al}_{0.2} \mathrm{Ga}_{0.8}\right)_{0.51} \mathrm{In}_{0.49}\right)_{0.5} \mathrm{P}_{0.5}$ barrier layer, lattice matched to GaAs, surrounds the QDs to enhance carrier confinement. A cladding layer consisting of $\left(\left(\mathrm{Al}_{0.55} \mathrm{Ga}_{0.45}\right)_{0.51} \mathrm{In}_{0.49}\right)_{0.5} \mathrm{P}_{0.5}$ completes the $\lambda$-cavity.

We have used $20 \% \mathrm{Al}$ in the barrier material, which results in a carrier confinement potential $\sim 90 \mathrm{meV}$ (for 
small QDs emitting high energetic X luminescence). Using higher $\mathrm{Al}$ contents in the barrier directly around the QD has turned out to be disadvantageous as it leads to an increased $\mathrm{Al}$ incorporation into the QD, thus resulting in a lowered carrier confinement potential. ${ }^{10,18}$

For the presented work, a circularly shaped MP with a diameter of $\sim 2.6 \mu \mathrm{m}$ was fabricated by focused ion beam (FIB) etching, providing lateral optical confinement and therefore highly directed emission to the top of the sample. A detailed description of the growth and FIB process can be found elsewhere. ${ }^{19}$

\section{MEASUREMENT SETUP}

To perform temperature-dependent $\mu$ PL-measurements, the sample was mounted in a He-flow cryostat equipped with a heating element to set the temperature of the cold finger with a precision of $\pm 0.2 \mathrm{~K}$ in a range of 4 to $130 \mathrm{~K}$. The QD was excited via a pulsed super-continuum fiber laser, selecting an excitation wavelength of $570 \mathrm{~nm}$ with an acousto-optic tunable filter, which provides a spectral bandwidth of $\Delta \lambda=3 \mathrm{~nm}$. The laser light is focused on the sample surface by a $50 \times$ objective resulting in an excitation spot diameter of $\sim 1.5 \mu \mathrm{m}$. The luminescence from the sample was collimated by the same objective and spectrally resolved by a $500 \mathrm{~mm}$ spectrometer with a 1200 lines/mm grating and an attached charge coupled device camera (1024 pixel columns), resulting in a spectral resolution better than $130 \mu \mathrm{eV}$.

Additionally, a Hanbury Brown and Twiss (HBT) setup was used to perform auto-correlation measurements. ${ }^{20}$ The spectrally pre-filtered emitted light was sent to a nonpolarizing 50/50 beam splitter cube (NPBS) and subsequently directed onto two avalanche photon diodes (APDs) one in each detection arm of the setup. The detection events of the APDs were temporally correlated to determine the $g^{2}(\tau)$ function over short timescales $\tau$ via photon statistics measurements, providing the possibility to verify SP emission.

\section{RESULTS}

In order to find energetically well separated QD luminescence lines, several MPs were investigated via $\mu \mathrm{PL}$ measurements. In contrast to the growth on exactly oriented substrates, the QD growth on miscut substrates leads to higher QD densities $\left(\sim 5.5 \times 10^{10}\right)$ (Refs. 11, 12, and 21$)$ but allows for better p-type doping and more disordered growth leading to a higher bandgap energy. ${ }^{22,23}$ Therefore, we have focused on small diameter MPs in order to reduce the number of optically excited QDs. Additionally we have used a sample area where the fundamental mode energy of the micropillar is detuned with respect to the QD ensemble luminescence maximum. In this spectral region, the separation of small dots was sufficiently large that single dots could be addressed. Due to the low number of QDs emitting in this energy region, the emission from the investigated MP showed no mode behavior.

The result of an excitation power-dependent measurement series of these QD emission lines are shown in Fig. 1(a).

At low excitation powers of $3 \mathrm{~W} / \mathrm{cm}^{2}$, the exciton emission is clearly dominant. Detailed analysis of the $\mathrm{X}$ and $\mathrm{XX}$ intensities show a super-linear increase of the biexciton intensity with increasing excitation power, leading to dominantly biexcitonic decay at high pump laser intensities above $\sim 150 \mathrm{~W} / \mathrm{cm}^{2}$. This typical power-dependence together with the XX binding energy of $\sim 7 \mathrm{meV}$ allows to clearly identify the $\mathrm{X}$ and $\mathrm{XX}$ decay channels. For higher pump powers $\geq 600 \mathrm{~W} / \mathrm{cm}^{2}$ we observe additional luminescence lines around the XX line, which can be attributed to multiexcitonic $^{24}$ and/or charged multi-excitonic transitions where additional carriers in higher shells of the QD are present. ${ }^{25}$

Figures 1(b) and 1(c) depict the temperature-dependence of the $\mathrm{X}$ and $\mathrm{XX}$ luminescence lines for a temperature range from $T=4 \mathrm{~K}$ to $120 \mathrm{~K}(130 \mathrm{~K})$ for power densities of $300 \mathrm{~W} / \mathrm{cm}^{2}\left(1500 \mathrm{~W} / \mathrm{cm}^{2}\right)$, respectively. In either case both lines reveal a pronounced redshift with increasing sample temperature, which reflects the gradual decrease of the barrier

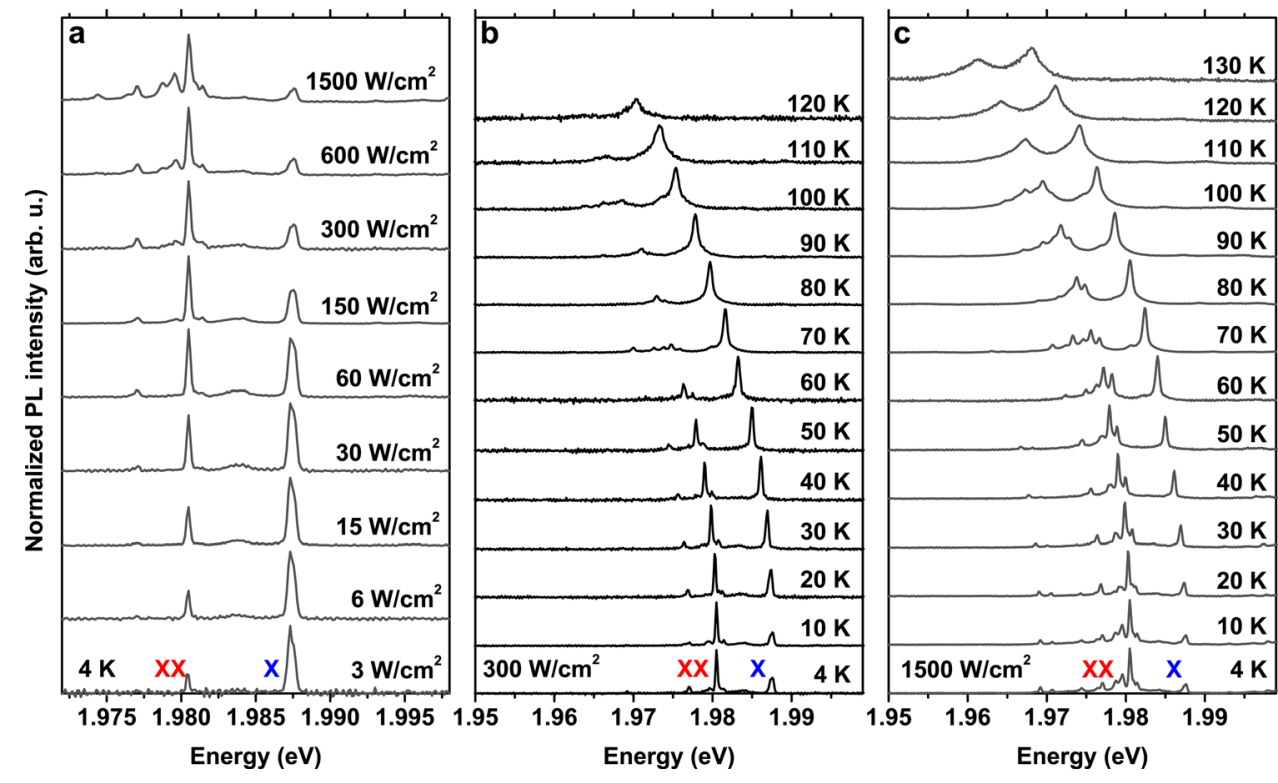

FIG. 1. (Color online) (a) Normalized power-dependent $\mu \mathrm{PL}$ spectra of a single QD measured at $4 \mathrm{~K}$ under pulsed excitation at $570 \mathrm{~nm}$. (b) Normalized temperature-dependent $\mu \mathrm{PL}$ spectra of the QD with an excitation power of $300 \mathrm{~W} / \mathrm{cm}^{2}$ under pulsed excitation at $570 \mathrm{~nm}$ (for the $\mathrm{X}$ and $\mathrm{XX}$ intensities and linewidth, see Fig. 2(b)). (c) Normalized temperature-dependent $\mu \mathrm{PL}$ spectra with an excitation power of $1500 \mathrm{~W} / \mathrm{cm}^{2}$ up to $130 \mathrm{~K}$. 

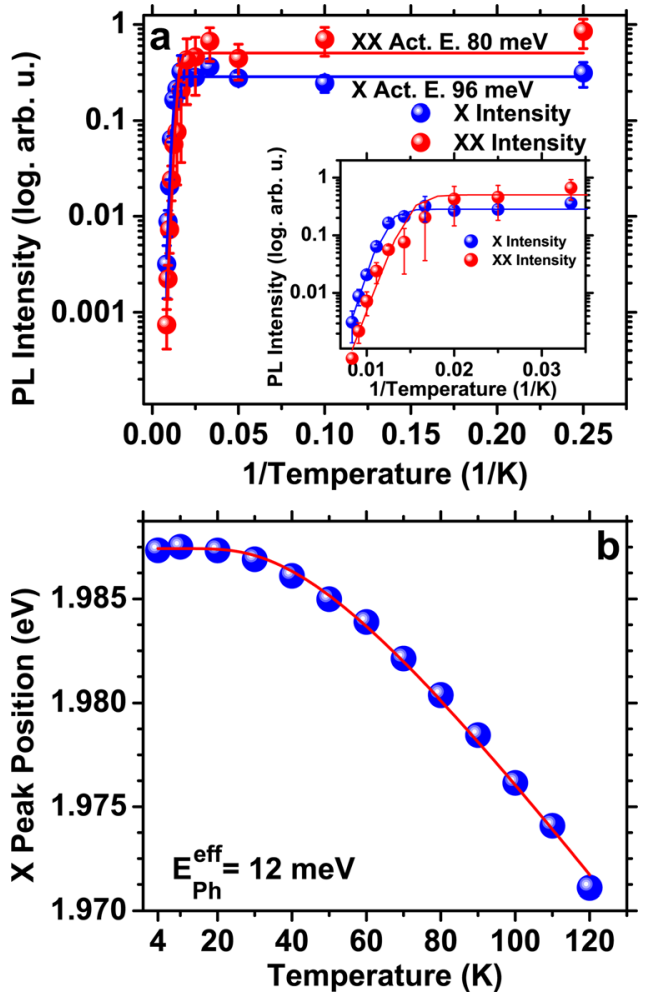

FIG. 2. (Color online) (a) Logarithmic $\mathrm{X}$ and $\mathrm{XX}$ emission intensity of Fig. 1(b) plotted against inverse temperature. Activation energy derived from Arrhenius fits: $E_{\mathrm{a}}^{\mathrm{X}}=96 \pm 7 \mathrm{meV}, E_{\mathrm{a}}^{\mathrm{XX}}=80 \pm 8 \mathrm{meV}$. (b) Temperature-dependent $\mathrm{X}$ peak position, with a fit revealing the effective phonon energy. Solid curves correspond to the models.

band-gap due to an phonon-induced increase of the mean lattice constant. $^{26}$

For this type of QDs, the effect of electron-hole pair trapping into an optically forbidden excitonic "dark" state has been demonstrated to have strong influence on the decay dynamics. ${ }^{27}$ Starting at low temperatures $(4 \mathrm{~K})$ under the conditions of moderate (Fig. 1(b)) or strong 1(c) excitation the XX decay signal is found to dominate the emission spectra. This can be interpreted as a consequence of the interplay of initial multi-carrier occupation in combination with subsequent "bright" X state depopulation into the competing "dark" state at $\sim 4 \mathrm{meV}$ lower energy. ${ }^{27}$ Consequently, for these conditions, the $\mathrm{X}$ luminescence is strongly suppressed.

At fixed excitation power but increasing temperature, we observe a gradual revival of the "bright" excitonic $\mu \mathrm{PL}$ intensity $I_{\mathrm{X}}$, where $I_{\mathrm{X}} / I_{\mathrm{XX}} \geq 1$ above $T=45 \pm 5 \mathrm{~K}$. We interpret this as a temperature-induced deliberation of trapped carriers back into the optically active $\mathrm{X}$ state by a spin-flip activation energy of $E_{\text {flip }}=k_{\mathrm{B}} T=3.9 \pm 0.5 \mathrm{meV}$.

The temperature-dependent XX and X emission intensities $I(T)$ have been individually analyzed by applying fits to each $\mu$ PL line in Fig. 1(b) based on an Arrhenius-type ${ }^{28}$ function

$$
I(T)=I_{0} \cdot\left[1+b \cdot \exp \left(-E_{\mathrm{a}} / k_{\mathrm{B}} T\right)\right]^{-1} .
$$

Here $I_{0}$ denotes the low temperature intensity limit, $b$ the carrier-phonon coupling strength, and $E_{\mathrm{a}}$ is the thermal activation energy for carrier escape from the QD (Fig. 2(a)). We have extracted values of $E_{\mathrm{a}}^{\mathrm{XX}}=80 \pm 8 \mathrm{meV}$ for the $\mathrm{XX}$ and
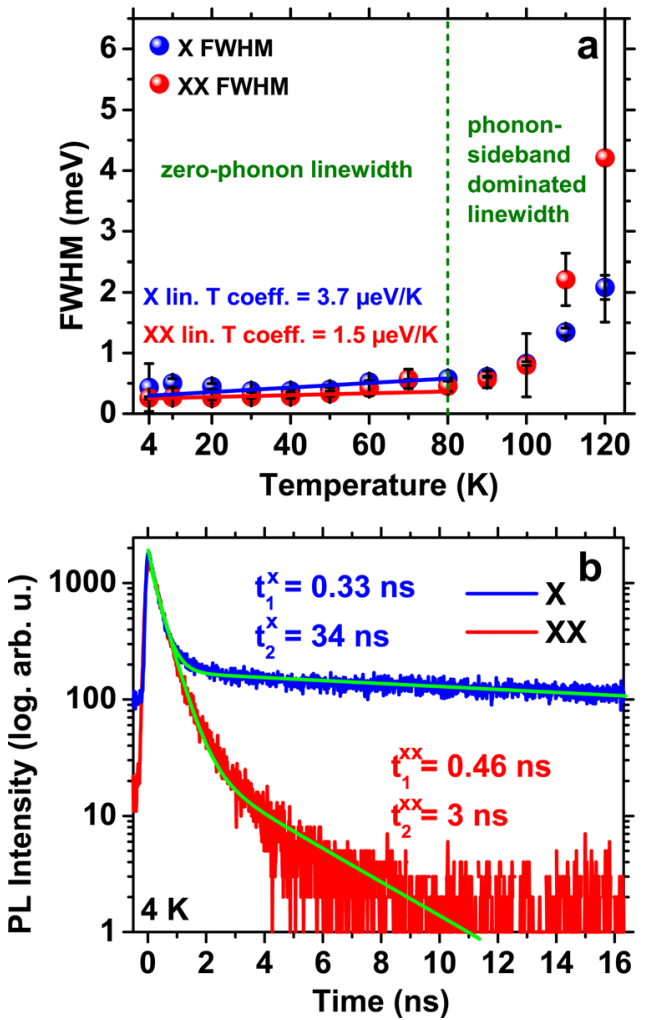

FIG. 3. (Color online) (a) Temperature-dependent FWHM of the X and XX lines of Fig. 1(b) under pulsed excitation with $300 \mathrm{~W} / \mathrm{cm}^{2}$. (b) Radiative decay time measurements of the $\mathrm{X}$ and $\mathrm{XX}$ luminescence lines at $4 \mathrm{~K}$.

$E_{\mathrm{a}}^{\mathrm{X}}=96 \pm 7 \mathrm{meV}$ for the $\mathrm{X}$ activation energy, which are in good agreement with the theoretical value of $\sim 90 \mathrm{meV}$ resulting from the barrier confinement at the given emission energy. ${ }^{10}$ The inset in Fig. 2(a) shows a zoom-in on the upper temperature range from 33 to $120 \mathrm{~K}$, clearly reflecting a stronger thermal quenching for the XX with respect to the $\mathrm{X}$.

The thermal redshift of the QD emission lines is displayed in Fig. 2(b). In order to extract the effective phonon energy $\left\langle E_{\mathrm{Ph}}\right\rangle$, responsible for the decrease of the barrier bandgap, the fit function ${ }^{26}$

$$
E(T)=E(0)-S\left\langle E_{\mathrm{ph}}\right\rangle\left[\left(\operatorname{coth}\left(\left\langle E_{\mathrm{ph}}\right\rangle /\left(2 k_{\mathrm{B}} T\right)\right)-1\right)\right]
$$

with $E(0)$ being the peak energy at the low temperature limit and $S$ a dimensionless coupling constant was applied to the data. From this analysis an effective phonon energy of $\left\langle E_{\mathrm{Ph}}\right\rangle=12 \pm 2 \mathrm{meV}$ could be deduced, which is in good agreement with previous measurements on similar types of QDs in bulk AlGaInP without micropillars. ${ }^{10}$

In the perspective of single-photon generation, an energetic overlap of the $\mathrm{X}$ and $\mathrm{XX}$ emission lines would have a negative impact on the photon statistics and signal purity. Therefore, the dependence of the $\mathrm{X}$ emission linewidth (FWHM) on temperature has been investigated, as shown in Fig. 3(a). The evolution of the zero-phonon FWHM for low temperatures $T \leq 80 \mathrm{~K}$ was fitted to the data in Fig. 1(b) according to ${ }^{29}$

$$
\Gamma(T)=\Gamma_{0}+a T
$$



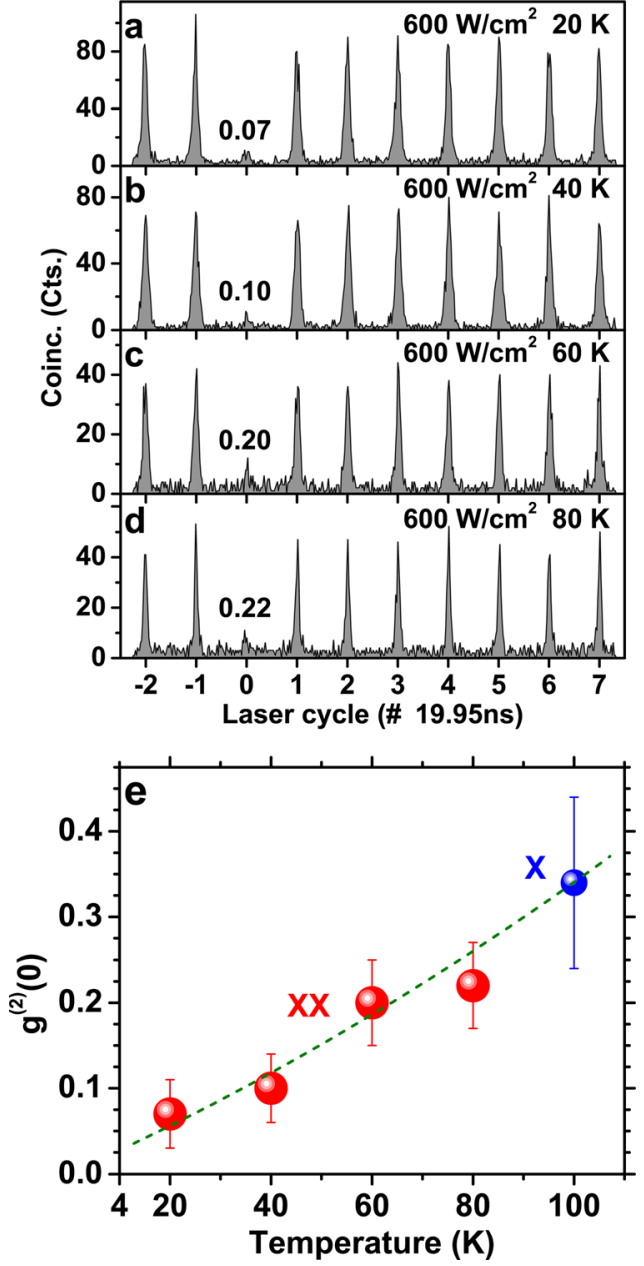

FIG. 4. (Color online) (a)-(d) Uncorrected auto-correlation histograms for the XX emission line at $20,40,60$, and $80 \mathrm{~K}$ at an excitation power of $600 \mathrm{~W} / \mathrm{cm}^{2}$. The numbers represent the normalized peak area of $7.98 \mathrm{~ns}$ resulting in $g^{2}(0)$ for the zero delay peak. (e) $g^{2}(0)$ temperature-dependence of the XX (red) and X (blue) emission line. The error bars represent $\pm \sigma$ of the standard deviation of the calculated Poisson level. (the green dashed line is a guide to the eye).

with the low temperature linewidth limit $\Gamma_{0} \approx 0.25 \mathrm{meV}$ and a linear temperature coefficient $a$, which describes the line broadening at low temperatures induced by dephasing due to acoustic phonon scattering. We want to note, that for temperatures above $\sim 80 \mathrm{~K}$ the $\mathrm{X}$ and $\mathrm{XX}$ linewidth is strongly influenced by the phonon sidebands and therefore rises abruptly. ${ }^{30}$

The linear temperature coefficients for the $\mathrm{X}$ and $\mathrm{XX}$ resulted in $a^{\mathrm{X}}=3.7 \pm 1 \mu \mathrm{eV}$ and $a^{\mathrm{XX}}=1.5 \pm 0.7 \mu \mathrm{eV}$, respectively, and are in good agreement with literature. ${ }^{29,31}$

From time-correlated single-photon counting (TCSPC) measurements performed on the $\mathrm{X}$ and $\mathrm{XX}$ emission lines at low temperatures, we observe biexponential radiative decay characteristics. As is depicted in Fig. 3(b), the $\mathrm{X}$ emission line reveals a short radiative time constant of $t_{1}^{\mathrm{X}}=330 \pm 2$ ps over the first order of magnitude, turning into significantly slower emission dynamics with $t_{2}^{\mathrm{X}}=33 \mathrm{~ns}$. In clear contrast, the XX radiative recombination reveals distinctly stronger decay with time constants of $t_{1}^{\mathrm{XX}}=460 \pm 10 \mathrm{ps}$ and $t_{2}^{\mathrm{XX}}=3 \pm 2 \mathrm{~ns}$, not showing any plateau-like behavior as for the $\mathrm{X}$ channel.
The observed behavior could be tentatively interpreted as a consequence of a long time scale feeding of the $\mathrm{X}$ "bright" state by spin flip from its "dark" state-in contrast to the fast decay of the XX where no such retarding mechanism via refilling from any "dark" state is expected.

In order to obtain high purity triggered single-photon emission, fast QD recombination dynamics with respect to the laser pulse excitation cycle is a necessary precondition. For the QD under study, only the XX decay is found to fulfill this requirement for low temperatures (see Fig. 3(b)).

Consequently, auto-correlation measurements have been performed on the XX emission line of the QD from 20 to $80 \mathrm{~K}$. The resulting histograms of the temporal correlation of photons for different temperatures are shown in Figs. 4(a)-4(d). For the calculation of $g^{2}(0)$ values, a constant low noise level mainly related to the detector dark counts has been subtracted from the raw correlation data, whereas a correction for uncorrelated (Poissonian) $\mu$ PL-signal background has not been performed. Figure 4(e) depicts the corresponding $g^{2}(0)$ values of the $\mathrm{XX}$ signal (red) derived from plots Figs. 4(a)-(d) and for the $\mathrm{X}$ emission line (blue) derived from Fig. 5(a). The observed increase of the $g^{2}(0)$ values toward higher temperatures is related to a rising uncorrelated $\mu \mathrm{PL}$ background.

Interestingly, for temperatures $T \geq 80 \mathrm{~K}$ the $\mathrm{XX}$ is found to significantly decrease in intensity, whereas the $\mathrm{X}$ decay becomes dominant and remains sufficiently strong for photon statistics measurements at even higher temperatures. For an auto-correlation measurement of the $\mathrm{X}$ emission line at moderate pump power of $600 \mathrm{~W} / \mathrm{cm}^{2}$ the spectrally selected energy range is depicted as a blue overlay in Fig. 5(b). The resulting coincidence data at $T=100 \mathrm{~K}$ are displayed in Fig. 5(a), showing clear sub-Poissonian photon statistics.
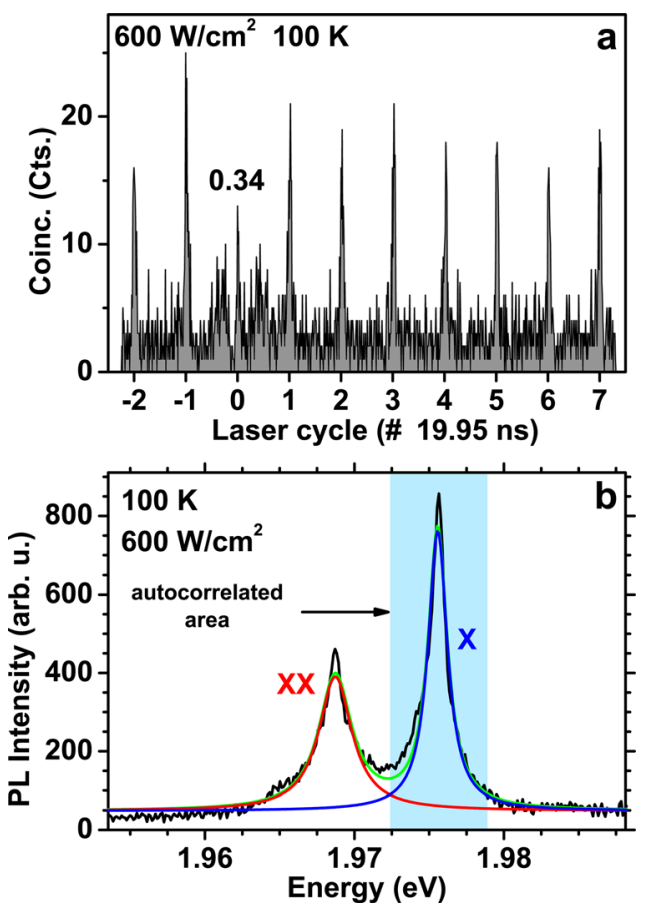

FIG. 5. (Color online) (a) Auto-correlation histogram for the $\mathrm{X}$ emission line at $100 \mathrm{~K}$. (b) $\mu \mathrm{PL}$ spectrum at $100 \mathrm{~K}$, indicating the autocorrelated area for the measurement above. 
In this case we additionally corrected the raw data for distinct $\mu \mathrm{PL}$ background in addition to the low detector noise. Accordingly, a signal to noise ratio of $\rho=S /(S+B)$ $=0.83 \pm 0.03$ has been obtained from the data in Fig. 5(b), where $S$ is the signal and $B$ the uncorrelated background. A correction for this background ${ }^{32}$ results in

$$
g_{\text {corr. }}^{(2)}(0)=g^{(2)}(0)-\left(1-\rho^{2}\right)=0.03 \pm 0.02,
$$

which indicates a very strong suppression of multi-photon events for the investigated QD.

We like to note that the effect of detector cross-talk artifacts in the data has been compensated by carefully choosing equally spaced binning areas of 7.98 ns symmetrically centered at the correlation peaks.

Within the measured temperature range, at moderate excitation power, the spectral overlap between the thermally broadened XX and $\mathrm{X}$ emission lines is still found to not strongly affect the purity of the emitted single-photons (see Fig. 1(b) and Fig. 5(b)).

\section{CONCLUSION}

In conclusion, detailed investigations on the emission characteristics of single $\mathrm{InP} /\left(\mathrm{Al}_{0.20} \mathrm{Ga}_{0.80}\right)_{0.51} \mathrm{In}_{0.49}$ quantum dots have been performed. The temperature-dependent evolution of the exciton linewidth and intensity were obtained. Exciton emission from the measured QD could be shown up to $130 \mathrm{~K}$, and triggered non-classical photon emission could be verified up to $100 \mathrm{~K}$ by auto-correlation measurements. High quality single-photon emission has additionally been demonstrated from biexcitonic decay under pulsed excitation up to $80 \mathrm{~K}$. Our measurements reveal self-assembled InP/ $\mathrm{AlGaInP}$ quantum dots as suitable emitters for the red spectral range between 650 and $710 \mathrm{~nm}$.

\section{ACKNOWLEDGMENTS}

We gratefully acknowledge funding from the $\mathrm{BMBF}$ via the EPHQUAM project.

${ }^{1}$ P. Michler, A. Kiraz, C. Becher, W. V. Schoenfeld, P. M. Petroff, L. Zhang, E. Hu, and A. Imamoglu, Science 290, 2282 (2000).

${ }^{2}$ C. Schneider, T. Heindel, A. Huggenberger, P. Weinmann, C. Kistner, M. Kamp, S. Reitzenstein, S. Höfling, and A. Forchel, Appl. Phys. Lett. 94, 111111 (2009).

${ }^{3}$ S. Strauf, N. G. Stoltz, M. T. Rakher, L. A. Coldren, P. M. Petroff, and D. Bouwmeester, Eur. Phys. J. D 1, 704 (2007).
${ }^{4}$ V. Zwiller, T. Aichele, W. Seifert, J. Persson, and O. Benson, Appl. Phys. Lett. 82, 1509 (2003).

${ }^{5}$ M. Pelton, C. Santori, G. S. Solomon, O. Benson, and Y. Yamamoto, Eur. Phys. J. D 18, 179 (2002).

${ }^{6}$ K. Sebald, P. Michler, T. Passow, D. Hommel, G. Bacher, and A. Forchel, Appl. Phys. Lett. 81, 2920 (2002).

${ }^{7}$ S. Kako, C. Santori, K. Hoshino, S. Götzinger, Y. Yamamoto, and Y. Arakawa, Nature Mater. 5, 887 (2006).

${ }^{8}$ R. P. Mirin, Appl. Phys. Lett. 84, 1260 (2004).

${ }^{9}$ A. Malko, D. Y. Oberli, M. H. Baier, E. Pelucchi, F. Michelini, K. F. Karlsson, M.-A. Dupertuis, and E. Kapon, Phys. Rev. B 72, 195332 (2005).

${ }^{10}$ W.-M. Schulz, R. Rossbach, M. Reischle, G. J. Beirne, M. Bommer, M. Jetter, and P. Michler, Phys. Rev. B 79, 035329 (2009).

${ }^{11}$ A. K. Nowak, E. Gallardo, D. Sarkar, D. Sanvitto, H. P. van der Meulen, J. M. Calleja, J. M. Ripalda, L. Gonzlez, and Y. Gonzlez, Physica E (Amsterdam) 42, 2509 (2010).

${ }^{12}$ A. K. Nowak, E. Gallardo, D. Sarkar, H. P. van der Meulen, J. M. Calleja, J. M. Ripalda, L. González, and Y. González, Phys. Rev. B 80, 161305 (2009).

${ }^{13}$ M. Reischle, C. Kessler, W.-M. Schulz, M. Eichfelder, R. Roßbach, M. Jetter, and P. Michler, Appl. Phys. Lett. 97, 143513 (2010).

${ }^{14}$ O. Thomas, Z. L. Yuan, J. F. Dynes, A. W. Sharpe, and A. J. Shields, Appl. Phys. Lett. 97, 031102 (2010).

${ }^{15}$ G. Beirne, M. Reischle, R. Roßbach, W.-M. Schulz, M. Jetter, J. Seebeck, P. Gartner, C. Gies, F. Jahnke, and P. Michler, Phys. Rev. B 75, 195302 (2007).

${ }^{16}$ R. Roßbach, M. Reischle, G. J. Beirne, M. Jetter, and P. Michler, Appl. Phys. Lett. 92, 071105 (2008).

${ }^{17}$ I. N. Stranski and L. Krastanow, Sitz. Ber. Akad. Wiss., Math.-Naturwiss. K1. Abt. IIb 146, 797 (1938).

${ }^{18}$ W.-M. Schulz, R. Roßbach, M. Reischle, G. J. Beirne, M. Jetter, and P. Michler, Phys. Status Solidi C 6, 906 (2009).

${ }^{19}$ W.-M. Schulz, T. Thomay, M. Eichfelder, M. Bommer, M. Wiesner, R. Roßbach, M. Jetter, R. Bratschitsch, A. Leitenstorfer, and P. Michler, Opt. Express 18, 12543 (2010).

${ }^{20}$ R. H. Brown and R. Twiss, Nature 177, 27 (1956).

${ }^{21}$ J. Persson, M. Holm, C. Pryor, D. Hessman, W. Seifert, L. Samuelson, and M.-E. Pistol, Phys. Rev. B 67, 035320 (2003).

${ }^{22}$ J. Lin, M.-J. Jou, C.-Y. Chen, and B.-J. Lee, J. Cryst. Growth 124, 415 (1992).

${ }^{23}$ J. Dong, S. J. Chua, Y.-J. Wang, and H.-R. Yuan, J. Cryst. Growth 269, 408 (2004).

${ }^{24}$ F. Findeis, A. Zrenner, G. Böhm, and G. Abstreiter, Solid State Commun. 114, 227 (2000).

${ }^{25}$ P. Hawrylak, Phys. Rev. B 60, 5597 (1999).

${ }^{26}$ K. P. O'Donnel and X. Chen, Appl. Phys. Lett. 58, 2924 (1991).

${ }^{27}$ M. Reischle, G. J. Beirne, R. Roßbach, M. Jetter, and P. Michler, Phys. Rev. Lett. 101, 146402 (2008).

${ }^{28}$ S. Arrhenius, Z. Phys. Chem. 4, 226 (1889).

${ }^{29}$ M. Bayer and A. Forchel, Phys. Rev. B 65, 041308(R) (2002).

${ }^{30}$ L. Besombes, K. Kheng, L. Marsal, and H. Mariette, Phys. Rev. B 63, 155307 (2001).

${ }^{31}$ G. Ortner, D. R. Yakovlev, M. Bayer, S. Rudin, T. L. Reinecke, S. Fafard, Z. Wasilewski, and A. Forchel, Phys. Rev. B 70, 201301 (2004).

${ }^{32}$ A. J. Shields, R. M. Stevenson, R. M. Thompson, Z. Yuan, B. E. Kardynal, "Generation of single photons using semiconductor quantum dots," NanoPhysics \& BioElectronics: A New Odyssey, edited by T. Chakraborty, F. Peeters, and U. Sivan (Elsevier, New York, 2002), Chap. 4, p. 1. 